\title{
Impact des Évolutions Climatiques sur les Ressources en eau des Petits Bassins en Afrique Sub-Saharienne: Application au Bassin Versant du Bandama à Tortiya (Nord Côte d'Ivoire)
}

\author{
Anzoumanan Kamagaté, Doctorant \\ Yao Blaise Koffi, Maître de Conférences
}

Laboratoire des Sciences et Techniques de l'Eau et de l'Environnement (LSTEE), Unité de Formation et de Recherche (UFR) des Sciences de la Terre et des Ressources Minières (STRM), Université Felix Houphouët Boigny, Abidjan

\section{Amani Michel Kouassi, Maître de conférences}

Institut National Polytechnique Felix Houphouët-Boigny (INP-HB), Département des Sciences de la Terre et des Ressources Minières (STeRMi), Laboratoire du Génie Civil, des Géosciences et des Sciences Géographiques, Yamoussoukro, Cote d'Ivoire

\section{Bernard Dje Kouakou, Docteur, SODEXAM}

Société d'Exploitation et de Développement Aéroportuaire Aéronautique et Météorologique (SODEXAM), Abidjan

\section{Diallo Seydou, Doctorant}

Laboratoire, Géosciences et Environnement, Unité de Formation et de réccherche des Sciences et Gestion de L'Envirnnement (UFR) des Sciences et Gestion de l'Environnemnt, Université Nangui Abrogoua (UNA), Abidjan

\section{Résumé}

L'objectif de cette étude est de montrer l'effet de l'évolution climatique sur les ressources en eau dans le bassin du Bandama à Tortiya. Pour atteindre cet objectif, diverses données (pluies, débits et températures) de sources différentes et méthodes (indices de NICHOLSON, filtre passe-bas d'ordre 2 de Hanning, test de PETTIT) ont été utilisées. L'application des indices de Nicholson associée au Filtre de Hanning aux séries pluviométriques et hydrologiques a mis en évidence une variabilité climatique caractérisée par une alternance de phases humides, normales et sèches. La méthode statistique de PETTIT indique une rupture pluviométrique à la station de Dikodougou et de Sirasso, intervenue respectivement en 1970 et en 1971. Ces ruptures marquent une modification 
des régimes pluviométriques et hydrologiques. Elles s'accompagnent d'une diminution de la pluviométrie de $9 \%$ à Sirasso et de $10 \%$ à Dikodougou. La baisse de la pluviométrie a eu pour effet une diminution des apports d'eau qui transitent dans les cours d'eau dans le bassin ainsi que les réserves en eau souterraines avec un déficit hydrologique de $66,5 \%$ des débits du fleuve Bandama à Tortiya. L'évaluation moyenne (1901-2016) de la recharge dans le bassin de Bandama à Tortiya calculée par la méthode de Thornthwaite sur les stations de Sirasso et de Dikodougou est de 197,5 mm d'eau infiltré soit un volume d'eau moyen annuel de $691250000 \mathrm{~m}^{3}$. Ainsi, L'alimentation en eau des aquifères du bassin versant du Bandama à Tortiya n'est pas très bien assurée à cause de la faiblesse des pluies depuis les années 70 .

Mots clés: Côte d'Ivoire, Bandama, Tortiya, climat, méthodes statistiques, ressources en eau 


\title{
Impact of Climate Change on the Water Resources of Small Basins in Sub-Saharan Africa: Application to the Bandama Watershed in Tortiya (North Ivory Coast)
}

\author{
Anzoumanan Kamagaté, Doctorant \\ Yao Blaise Koffi, Maître de Conférences
}

Laboratoire des Sciences et Techniques de l'Eau et de l'Environnement (LSTEE), Unité de Formation et de Recherche (UFR) des Sciences de la Terre et des Ressources Minières (STRM), Université Felix Houphouët Boigny, Abidjan

Amani Michel Kouassi, Maître de conférences

Institut National Polytechnique Felix Houphouët-Boigny (INP-HB), Département des Sciences de la Terre et des Ressources Minières (STeRMi), Laboratoire du Génie Civil, des Géosciences et des Sciences Géographiques, Yamoussoukro, Cote d'Ivoire

Bernard Dje Kouakou, Docteur, SODEXAM

Société d'Exploitation et de Développement Aéroportuaire Aéronautique et Météorologique (SODEXAM), Abidjan

\section{Diallo Seydou, Doctorant}

Laboratoire, Géosciences et Environnement, Unité de Formation et de réccherche des Sciences et Gestion de L'Envirnnement (UFR) des Sciences et Gestion de l'Environnemnt, Université Nangui Abrogoua (UNA), Abidjan

\section{Abstract}

The objective of this study is to show the effect of climate change on water resources in the Bandama Basin in Tortiya. To achieve this objective, various data (rainfall, flow rates, temperatures) from different sources and methods (NICHOLSON indices, Hanning order 2 low pass filter, PETTIT test) were used. The application of Nicholson indices associated with the Hanning Filter to the rainfall and hydrological series revealed a climatic variability characterized by an alternation of wet, normal and dry phases. The statistical method of PETTIT indicates a rainfall rupture at the Dikodougou and Sirasso stations, which occurred in 1970 and 1971, respectively. These ruptures mark a modification of rainfall and hydrological regimes. They are accompanied by a decrease in rainfall of $9 \%$ in Sirasso and $10 \%$ in Dikodougou. The drop in rainfall has resulted in a decrease in the inflow of 
water that passes through the watercourses in the basin as well as the groundwater reserves with a hydrological deficit of $66.5 \%$ of flows from Bandama to Tortiya. The average assessment of recharge in the Bandama Basin in Tortiya calculated by the Thornthwaite method on the Sirasso and Dikodougou stations is $197.5 \mathrm{~mm}$ of infiltrated water, ie an average volume of water of $691,250,000 \mathrm{~m} 3$. Thus, the water supply of the aquifers of the Bandama sub-basin in Tortiya is not very well assured because of the weak rains since the 70 's.

Keywords: Côte d'Ivoire, Bou, statistical methods, water resource, news climate

\section{Introduction}

L'importance de l'eau pour la vie sur terre et pour les activités de l'homme en général fait que scientifiques et gestionnaires s'inquiètent désormais des conséquences des changements du climat sur le cycle hydrologique, la disponibilité et la qualité des eaux souterraines et des eaux de surface. En effet, plusieurs travaux (Paturel et al.,1996 et FAO 2005) ont confirmé, la relation entre le climat et la disponibilité des ressources en eau. Ces auteurs ont montré un impact marqué des déficits pluviométriques des dernières décennies sur les écoulements des cours d'eau. De façon globale, ces auteurs ont caractérisé la variabilité climatique et leurs impacts sur la disponibilité des ressources en eau. Au-delà de la variabilité spatio-temporelle permanente qu'ils mettent en exergue, selon le Groupe d'experts intergouvernemental sur l'évolution du climat GIEC (2007), un climat modifié caractérisé par l'élévation de la température moyenne du globe due à l'augmentation de la concentration du $\mathrm{CO}_{2}$ dans l'atmosphère, induira une amplification des phénomènes extrêmes (cyclones, sécheresse, inondations, etc.).

Ainsi, la variabilité du climat en Afrique de l'Ouest et Centrale en général et en Côte d'Ivoire en particulier, n'est plus à démontrer (Kouassi et al., 2008, Soro et al., 2011 ; Noufé, 2011 ; Soro, 2014, Traoré, 2016; Amani et al., 2017). En Côte d'Ivoire, elle a d'abord affecté le Nord, puis progressivement s'est étendue vers le centre et enfin sur le littoral. Ces anomalies pluviométriques constatées depuis près de quatre décennies ont connu une résonance exceptionnelle dans les régions nord (Poro) et centre du pays. Mais en réalité, l'ensemble du pays présente une vulnérabilité importante aux déficits pluviométriques. Cette situation a pour conséquence la baisse des débits des cours d'eau.

Ces différentes études faites, restent cependant à l'échelle du grand bassin et cela contribue à "gommer" certaines particularités locales. Cette étude vient donc corriger cette insuffisance en réduisant l'échelle pour mieux 
apprécier l'impact de la variabilité climatique sur les ressources en eau d'un petit sous bassin versant du Bandama. C'est dans cette perspective que s'inscrit cette étude.

L'objectif principal de cet article est de caractériser l'impact de l'évolution climatique sur les ressources en eau (eau de surface et souterraine) sur le bassin versant du Bandama à Tortiya. De façon spécifique, il s'agit de caractériser la variabilité climatique et analyser ses impacts sur les ressources en eau du bassin versant du Bandama à Tortiya.

\section{Cadre de l'étude}

Le bassin versant du Bandama à Tortiya constitue la zone retenue pour cette étude (Figure 1). Il est situé à l'extrême Sud de la région du Poro au Nord de la Côte d'Ivoire, entre les longitudes $5^{\circ} 30^{\prime}$ 'et $6^{\circ} 15^{\prime}$ ' Ouest et les latitudes $8^{\circ} 30^{\prime}$ et $9^{\circ} 30^{\prime}$ Nord. Il s'étend sur une superficie de $3500 \mathrm{~km}^{2}$. Le bassin est drainé par l'un des plus grands affluents du Bandama le Bou.

Le climat de la zone appartient au régime tropical de type soudanosahélien dont le rythme des saisons est réglé par le déplacement du Front Intertropical (FIT). La saison des pluies est unique et dure 7 mois. Elle est continue et s'étend d'avril à octobre (Jourda, 2005). Le maximum des précipitations se situe en août et septembre. La pluviométrie moyenne annuelle de la région est estimée à environ $1200 \mathrm{~mm}$ (Jourda, 2005).

La végétation du bassin est essentiellement savanicole. Il s'agit de savanes herbeuses, arbustives ou arborées. Des forêts galeries et des forêts claires sont aussi rencontrées. Ces formations couvrent respectivement $10 \%$, $30 \%, 2 \%$ et $10 \%$ du paysage, dont le reste est occupé par les sols nus et les cultures (Poilecot et al., 1991). La grande faune est encore présente, mais en quantité réduite et se rencontre surtout dans les forêts classées.

Au plan géologique le bassin s'inscrit dans l'histoire du craton Ouest africain (Savané, 1997; Djro, 1998 ; Soro, 2002 ; Touré, 2007). Deux grands ensembles peuvent être distingués sur le plan lithologique: Les formations Birrimiennes (roches volcaniques, volcano-sédimentaires et sédimentaires, métamorphisées déposées dans les sillons intracratoniques) et les formations granitoïdes éburnéennes (massifs granitiques au sein desquels on y distingue plusieurs générations de granites et les migmatites).

Sur le plan hydrogéologique, deux types d'aquifères sont présents : les réservoirs d'altérites et les réservoirs de fissures qui offrent des ressources en eau pérennes liées au réseau souterrain de fractures très développé dans ces formations du fait de la tectonique polyphasée ( Savané, 1997; Jourda, 2005). Dans l'ensemble, le relief du bassin du Bandama à Tortiya est plat et monotone, mais quelques sommets se distinguent, où les altitudes varient de 185 à 568 m. 


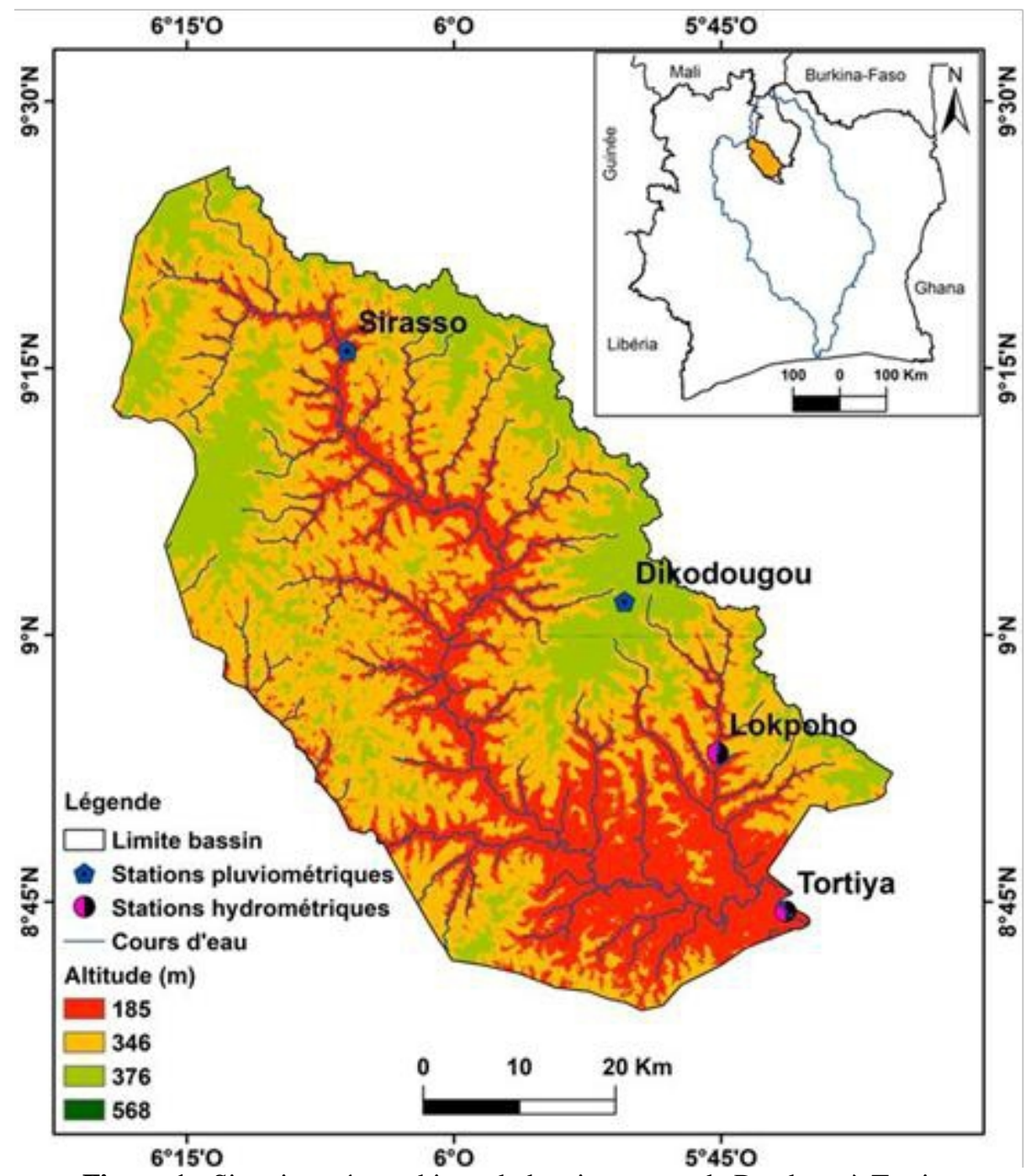

Figure 1 : Situation géographique du bassin versant de Bandama à Tortiya

\section{Données et Méthodes}

\subsection{Données de l'étude}

\subsubsection{Données hydroclimatiques}

Les données hydroclimatiques proviennent de la base de données du CRU (Climate Research Unit). Il s'agit des hauteurs mensuelles des pluies P $(\mathrm{mm})$, des températures moyennes de l'air $\mathrm{T}\left({ }^{\circ} \mathrm{C}\right)$, et des évapotranspirations potentielles (mm). Elles s'étendent sur la période de 1901 à 2016. Ces données ont été choisies car elles ont fait l'objet de plusieurs études et ont abouti à des résultats assez satisfaisants. Ainsi, les travaux d'Assoma, (2013) dans le bassin versant de l'Agneby ont montré une forte corrélation entre les données de la SODEXAM (Société d'Exploitation et de Développement Aéroportuaire 
Aéronautique et Météorologique) et les données CRU. A bordant dans le même sens Nka, (2016) a aussi montré une forte corrélation entre les données de plusieurs stations pluviométriques de l'Afrique de l'Ouest et celles de CRU.

\subsubsection{Données hydrométriques}

Les données hydrométriques ont été mises à notre disposition par la Direction Générale des Infrastructures de l'Hydraulique Humaine (DGIHH). Ces données concernent les débits journaliers des principaux cours d'eau du bassin. Le réseau hydrométrique pour cette étude est constitué de 02 stations hydrométriques. Ces stations sont celles de Tortiya et de Lokpoho. Les données couvrent la période de 1975 à 2002 pour le Lokpoho et de 1962 à 2013 pour Tortiya. Elles comportent des lacunes qui vont de quelques mois à une année entière (Tableau 1).

Tableau 1 : Caractéristiques des stations hydrométriques

\begin{tabular}{|l|l|l|l|l|}
\hline $\begin{array}{l}\text { Stations } \\
\text { hydrologiques }\end{array}$ & $\begin{array}{l}\text { Surface des } \\
\text { sous- } \\
\text { bassins } \\
\left(\mathrm{km}^{2}\right)\end{array}$ & $\begin{array}{l}\text { Période } \\
\text { d'observation }\end{array}$ & $\begin{array}{l}\text { Nombre d'années } \\
\text { d'observation }\end{array}$ & $\begin{array}{l}\text { Nombre } \\
\text { d'années } \\
\text { avec lacune }\end{array}$ \\
\hline Lokpoho & 772 & $1975-2002$ & 28 & 09 \\
\hline Tortiya & 3500 & $1962-2013$ & 52 & 05 \\
\hline
\end{tabular}

Les valeurs manquantes au sein des séries chronologiques de débit moyen annuel ont été déterminées par la méthode du critère de proportionnalité analogue. Ainsi, les différentes surfaces des sous-bassins ont été calculés et les rapports ci-dessous ont été déterminés et utilisés:

$$
\frac{Q_{\text {Lokpoho }}}{S_{\text {Lokpoho }}}=\frac{Q_{\text {Tortiya }}}{S_{\text {Tortiya }}} Q \text { Tortiya }=4,534 . Q_{\text {Lokpoho }}
$$

Les périodes d'observations hydrométriques sont moins longues que celles des données pluviométriques.

\subsection{Méthodes}

\subsubsection{Caractérisation du climat}

La pluviométrie est un paramètre principal pour caractériser le climat (Chuzeville, 1990). La méthodologie qui a été adoptée pour caractériser le climat s'articule autour de la méthode des indices de NICHOLSON, du Filtre non récursif passe-bas d'ordre 2 de Hanning, de la méthode statistique de PETTIT et de la méthode de détermination des déficits hydroclimatiques. Ces différentes méthodes ont été utilisées par plusieurs auteurs en Afrique et en particulier en Côte d'Ivoire (Kanohin et al., 2009; Soro et al., 2011; Kouassi et al., 2012; Soro, 2014; Traoré, 2016; Amani et al., 2017) pour la caractérisation du climat et ont donné de bons résultats. 
Pour l'application du test de Pettitt, recours a été fait au logiciel Khronostat qui est un programme de calcul mis au point par HydroSciences Montpellier.

\subsubsection{Caractérisation de l'impact du climat sur les eaux de surface}

L'impact de la variabilité climatique sur les eaux de surface est évalué par le calcul des coefficients de tarissement et des volumes d'eau mobilisés par les aquifères. Selon Saley (2003), le coefficient de tarissement permet d'apprécier l'état des « entrées » d'eau qui contribueraient à la modification apparente des relations pluie/débit observées sur les bassins fluviaux. Il permet ainsi de comparer l'évolution de l'eau de surface et le stockage de l'aquifère afin de mieux cerner le comportement du réservoir de vidange.

Il a été déterminé à l'aide de la méthode dichotomique proposée par Savané et al., (2003). Quant au volume d'eau mobilisé par l'ensemble des aquifères, il a été obtenu à partir d'une intégration de la formule de l'équation sur l'intervalle 0 à $+\infty$

$$
V_{\text {mob }}=\int_{0}^{+\infty} Q_{0} e^{-k t}=\frac{86400 * Q_{0}}{k}
$$

avec : Q0 = débit initial (débit au début du tarissement), exprimé en $\mathrm{m}^{3} / \mathrm{s}$; $\mathrm{k}=$ coefficient de tarissement de Maillet, exprimé en jours ${ }^{-1}$ ).

\subsubsection{Caractérisation de l'impact du climat sur les eaux souterraines}

La caractérisation de l'impact du climat sur les eaux souterraines est basée sur le calcul du bilan hydrologique à partir de la méthode de Thornthwaite compte tenu des données disponibles que sont la température et la pluviométrie. Les principaux paramètres recherchés sont l'évapotranspiration potentielle (ETP), l'évapotranspiration réelle (ETR) et l'excédent (Exc.) ou infiltration efficace. Le calcul du bilan hydrologique est appliqué à la station de Sirasso et de Dikodougou à partir de 1901, date à laquelle les mesures de températures sont disponibles.

\section{Résultats}

\subsection{Impact de la variabilité climatique dans le bassin du Bandama à} Tortiya

\subsubsection{Analyses des indices pluviométriques}

L'évolution des indices de Nicholson (variable centrée réduite) associée au Filtre de Hanning montre que trois grandes périodes climatiques ont marquées la variabilité pluviométrique sur l'ensemble du bassin versant de Bandama à Tortiya (Figure 2). 

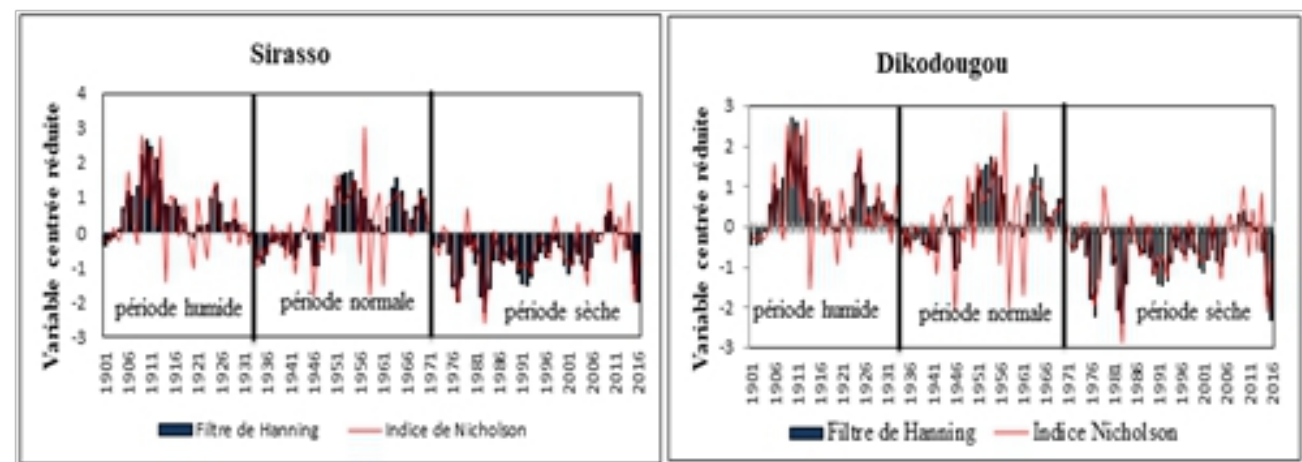

Figure 2 : Variabilité interannuelle de la pluviométrie dans le bassin versant de Bandama à Tortiya (de 1901 à 2016)

L'analyse de la figure 2 montre que l'évolution interannuelle de la pluviométrie à Sirasso se caractérise par une période humide de 1901 à 1930 avec une moyenne de $1433 \mathrm{~mm}$, une période normale de 1931 à 1971 avec une moyenne de $1385 \mathrm{~mm}$ et une période sèche de 1972 à 2016 avec une moyenne de $1266 \mathrm{~mm}$. La période humide comporte une année remarquable 1957 avec la plus forte pluviométrie évaluée à 1884,6 mm, soit une hausse de 491,6 mm. Les années 1977, 1983, 2005 et 2015 sont les plus déficitaires au cours de la période sèche avec respectivement 1002,9 mm, 893,5 mm, $1088 \mathrm{~mm}$ et 1005 $\mathrm{mm}$.

A la station de Dikodougou, la fluctuation interannuelle est caractérisée par une période humide de 1901 à 1932 avec une moyenne de $1391 \mathrm{~mm}$, une période normale de 1933 à 1970 avec une moyenne de 1348 mm et une période sèche de 1971 à 2016 avec une moyenne de 1224 mm. L'année remarquable de la période humide est 1913 avec 1795,5 mm de pluie soit un excédent de $404,5 \mathrm{~mm}$ par rapport à la moyenne interannuelle qui s'élève à $1391 \mathrm{~mm}$. Les années 19771983 et 2015 sont les plus déficitaires au cours de la période sèche avec respectivement $961,8 \mathrm{~mm}, 793,7 \mathrm{~mm}$ et 932,8 $\mathrm{mm}$.

Après avoir déterminé les périodes humides, normales et sèches par les indices de Hanning et de Nicholson, l'application du test de rupture de Pettitt a permis de situer les périodes de rupture au sein des séries chronologiques.

\subsubsection{Rupture climatique et des déficits pluviométriques}

L'analyse des résultats consignés dans le tableau 2 indique une rupture en 1971 au seuil de confiance de $95 \%$ pour la station de Sirasso et une rupture en 1970 au seuil de $99 \%$ pour la station de Dikodougou. La probabilité de dépassement associée au test de Pettitt montre que les ruptures constatées sur l'ensemble des stations du bassin, sont très significatives (seuils inférieures à $1 \%)$. 
L'ampleur de la baisse pluviométrie observée sur les deux (02) stations du bassin a été quantifiée par le calcul des déficits dus aux ruptures détectées dans les séries de données. Les déficits pluviométriques varient de $9 \%$ (Sirasso) à $10 \%$ (Dikodougou) (Tableau 3). Cette gamme de variation indique que la sécheresse revêt un caractère globalement modéré sur le bassin versant du Bandama à Tortiya.

Tableau 2 : Rupture climatique obtenu avec le test de Pettitt

\begin{tabular}{lllll}
\hline Stations & $\begin{array}{l}\text { Longueur } \\
\text { de la série } \\
\text { en années }\end{array}$ & $\begin{array}{l}\text { Années de } \\
\text { rupture }\end{array}$ & $\begin{array}{l}\text { Probabilité de } \\
\text { dépassement } \\
(\boldsymbol{\%})\end{array}$ & $\begin{array}{l}\text { Niveau de } \\
\text { signification }\end{array}$ \\
\hline Sirasso & 115 & 1971 & $2,41.10^{-4}$ & $\begin{array}{l}\text { Rupture très } \\
\text { significative }\end{array}$ \\
\hline Dikodougou & 115 & 1970 & $1,38.10^{-4}$ & $\begin{array}{l}\text { Rupture très } \\
\text { significative }\end{array}$ \\
\hline
\end{tabular}

Tableau 3 : Déficits pluviométriques des stations de Sirasso et de Dikodougou

\begin{tabular}{|c|c|c|c|c|c|}
\hline Stations & $\begin{array}{c}\text { Période } \\
\text { d'étude }\end{array}$ & Référence & $\begin{array}{c}\text { P moy avant } \\
\text { Rupture } \\
\text { (mm) }\end{array}$ & $\begin{array}{c}\text { P moy après } \\
\text { Rupture } \\
\text { (mm) }\end{array}$ & $\begin{array}{c}\text { Déficit } \\
\text { (\%) }\end{array}$ \\
\hline Sirasso & $1901-2016$ & 1971 & 1406 & 1266 & -9 \\
\hline Dikodougou & $1901-2016$ & 1970 & 1367 & 1224 & -10 \\
\hline
\end{tabular}

\subsubsection{Variabilité saisonnière des pluies avant et après rupture}

Les fluctuations mensuelles de la pluviométrie avant et après la rupture ont été analysées (Figure 3) afin de mieux percevoir les variations. En effet, la variabilité saisonnière de la pluviométrie a des conséquences importantes sur les cultures pluviales, du fait que l'agriculture dans le bassin est encore basée sur les systèmes agraires pluviaux et donc toujours tributaire des aléas climatiques, rendant la production incertaine. L'observation des graphes montre que la distribution des précipitations mensuelles avant et après rupture est similaire. Quelque soit la station considérée, on remarque que les hauteurs mensuelles de pluie ont baissé pendant tous les mois de l'année après rupture. Dans l'ensemble, la succession des saisons ne connaît pas de changement significatif, mais la différence notable réside dans le fait que les quantités d'eau précipitées après la rupture sont relativement en baisse quelque soit les saisons.

Le mois d'août demeure le plus arrosé sur les deux (02) stations avant et après rupture. Toutefois, les mois d'août et de septembre sont les plus affectés par cette baisse pluviométrique. Les mois secs durant lesquels quelques précipitations isolées sont constatées, sont plus ou moins affectés par la baisse de la pluviométrie. 


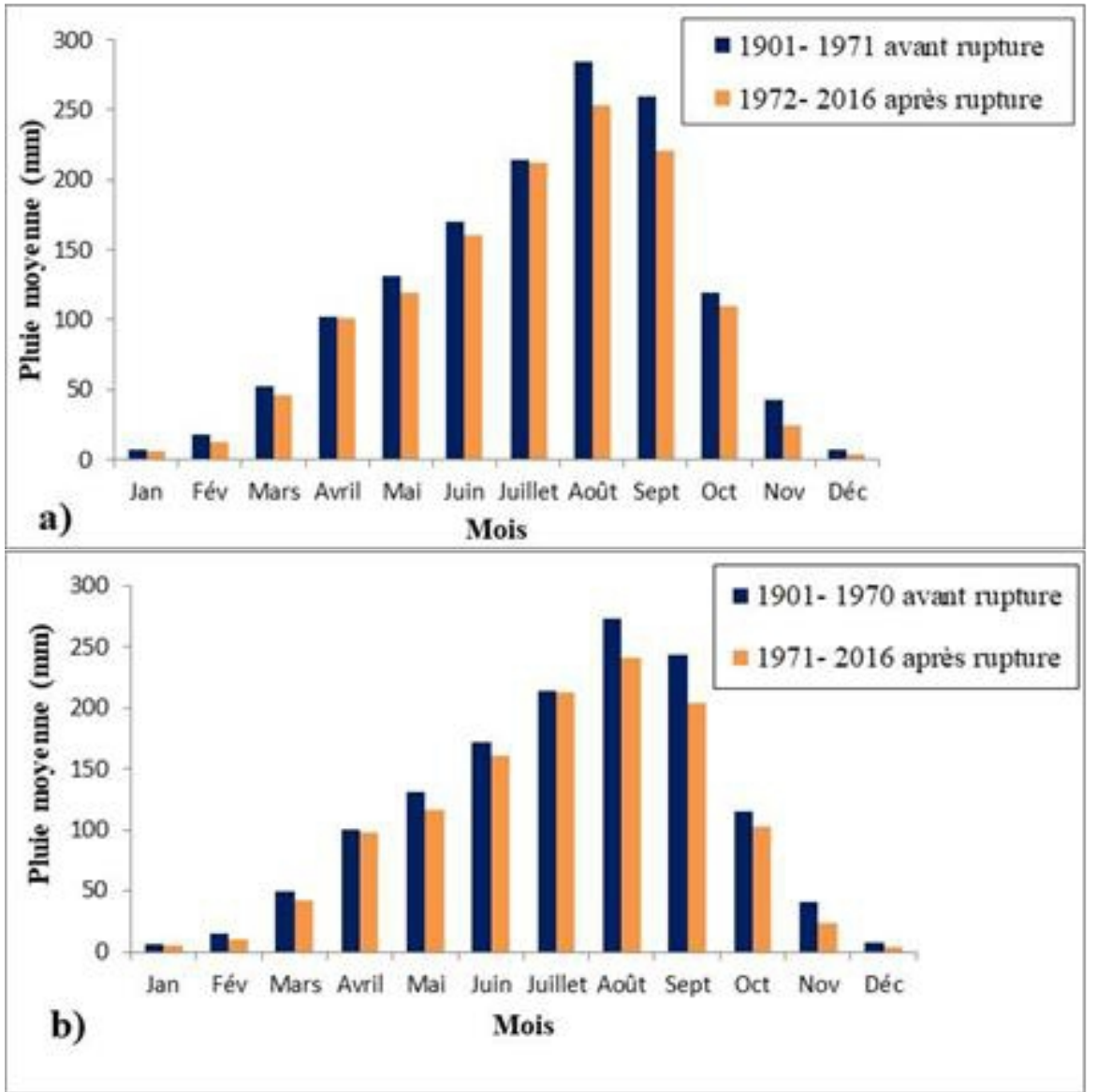

Figure 3: Variation moyenne mensuelle interannuelle des précipitations avant et après rupture : a) Sirasso ; b) Dikodougou

\subsection{Impact du climat sur les eaux de surface du bassin versant de Bou}

\subsubsection{Variation interannuelle des indices pluviométriques et} hydrométriques

La station de Tortiya a été choisie pour suivre la variabilité hydrologique du bassin pour la longueur de sa série. L'analyse de la figure 4 montre une corrélation parfaite entre les indices pluviométriques et hydrométriques à la station de Tortiya. Cette tendance synchrone de la relation pluie-débit est exprimée par l'augmentation des débits en période de pluviométrie excédentaire (1962-1971) et par la baisse des débits en période de déficit pluviométrique (1972-2013). 


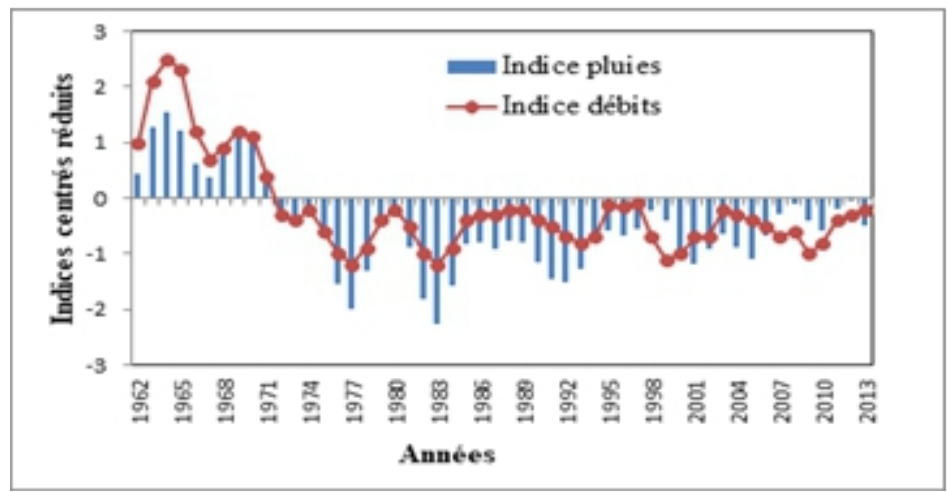

Figure 4: Variations interannuelles des indices centrés réduits de débits et de pluies à la station de Tortiya (1962-2013)

Les valeurs minimales d'indice de pluies et de débits sont observées en 1977, 1982 et 1983. Ces différentes périodes sont la résultante de plusieurs années pluviométriques déficitaires observées depuis 1971. En somme, la variabilité pluviométrique semble influencer le processus de production des écoulements.

Le déficit d'écoulement calculé de part et d'autre de la date de rupture (1971) dans la série chronologique des débits annuels sur la période 19622013, est de 66,5\% (Tableau 4 ). Les années 1977 et 1983 sont les plus déficitaires avec des modules annuels respectifs de $21,1 \mathrm{~m}^{3} / \mathrm{s}$ et $6,1 \mathrm{~m}^{3} / \mathrm{s}$.

Tableau 4 : Déficits d'écoulement de la station de Tortiya (1962-2013)

\begin{tabular}{|c|c|c|c|c|c|c|}
\hline \multicolumn{3}{|c|}{ Avant rupture $\left(\mathrm{m}^{3} / \mathrm{s}\right)$} & \multicolumn{3}{c|}{ Après rupture $\left(\mathrm{m}^{3} / \mathrm{s}\right)$} \\
\hline Stations & Référence & Moyenne & Ecart type & Moyenne & Ecart type & $\begin{array}{c}\text { Déficit } \\
(\%)\end{array}$ \\
\hline Tortiya & 1971 & 144,2 & 25,7 & 47,2 & 22,2 & $-66,5$ \\
\hline
\end{tabular}

\subsubsection{Variation des coefficients de tarissement}

Le coefficient de tarissement moyen a été déterminé avant et après la rupture de 1971, au cours de la période 1962-2013 à la station de Tortiya. Les débits moyens journaliers ont été calculés sur les périodes 1962-1971 et 19722013. Ces valeurs ont permis la détermination des coefficients de tarissement à cette station (Tableau 5). L'analyse du tableau montre une faible augmentation du coefficient de tarissement après la rupture. Cela pourrait s'expliquer par la baisse de la pluviométrie au cours de cette période.

Tableau 5 : Coefficients de tarissement interannuels autour de la rupture de 1971

\begin{tabular}{|c|c|c|}
\hline \multirow{2}{*}{ Station } & Période & $\begin{array}{c}\text { Coefficient de tarissement } \\
\left(\mathrm{j}^{-1}\right)\end{array}$ \\
\hline \multirow{2}{*}{ Tortiya } & $1962-1971$ & $3,333.10^{-2} / \mathrm{j}$ \\
\cline { 2 - 3 } & $1972-2013$ & $3,361.10^{-2} / \mathrm{j}$ \\
\hline
\end{tabular}

Les figures 5 et 6 sont les courbes de tarissement obtenues avant et après la rupture de 1971 à la station de Tortiya. L'analyse des courbes avant 
et après la rupture montre que le débit décroit rapidement. Il est passé de $303,73 \mathrm{~m}^{3} / \mathrm{s}$ à $96,91 \mathrm{~m}^{3} / \mathrm{s}$ en l'espace de 40 jours avant la rupture et de 120,8 $\mathrm{m}^{3} / \mathrm{s}$ à $28,97 \mathrm{~m}^{3} / \mathrm{s}$ en espace de 50 jours après la rupture. Les valeurs du coefficient de tarissement se situent entre $2,36.10^{-2} / \mathrm{j}$ et $4,57.10^{-2} / \mathrm{j}$ avant la rupture et entre $1,98 \cdot 10^{-2} / \mathrm{j}$ et $5,31.10^{-2} / \mathrm{j}$ après la rupture.
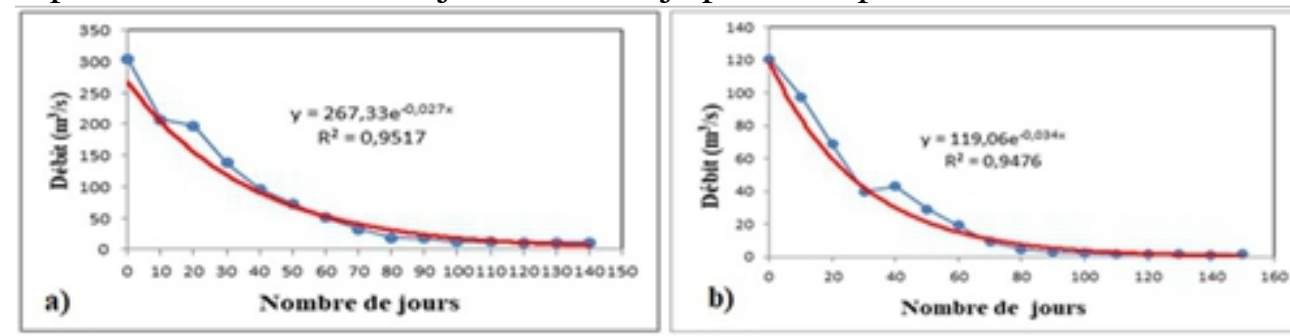

Figure 5 : Courbe de tarissement à la station de Tortiya

a) avant la rupture b) après la rupture

\subsubsection{Variations des volumes mobilisés par les aquifères}

Les volumes d'eau mobilisés par les aquifères sur la période 19622013 , sont compris entre 0,027 et $1,598 \mathrm{~km}^{3}$, avec une moyenne de $0,572 \mathrm{~km}^{3}$. De 1962 jusqu'à la rupture hydrologique en 1971, le volume moyen d'eau mobilisé par les aquifères s'élève à $0,840 \mathrm{~km}^{3}$ (Tableau. 6) pour un coefficient de tarissement moyen de 3,333.10-2/ j. Après la rupture, de 1972 à 2013, ce volume moyen est estimé à $0,508 \mathrm{~km}^{3}$ pour un coefficient de tarissement moyen de $3,361.10^{-2} / \mathrm{j}$, soit une baisse de $24,6 \%$.

Tableau 6 : Volume d'eau mobilisé par les aquifères avant et après la rupture

\begin{tabular}{|c|c|c|}
\hline Station & Période & Volumes mobilisés $\left(\mathrm{Km}^{3}\right)$ \\
\hline Tortiya & $1962-1971$ & 0,840 \\
\cline { 2 - 3 } & $1972-2013$ & 0,508 \\
\hline
\end{tabular}

L'analyse de la figure 7 montre une variation importante de la lame d'eau écoulée avec une tendance à la baisse intervenue après les années 1970. Les valeurs maximales et minimales obtenues sont respectivement de $776 \mathrm{~mm}$ en 1964 et $19 \mathrm{~mm}$ en 2000. Quant aux coefficients de tarissement, ils connaissent une augmentation depuis les années 1970. Les plus fortes valeurs de coefficient de tarissement sont obtenues en $1977\left(4,295.10^{-2} / \mathrm{j}\right), 1983$ $\left(5,310.10^{-2} / \mathrm{j}\right)$ et $1999\left(4,318 \cdot 10^{-2} / \mathrm{j}\right)$.

Le déficit pluviométrique a occasionné une augmentation des coefficients de tarissement et une baisse des volumes d'eau mobilisés par les aquifères. Il est donc nécessaire d'évaluer les impacts des fluctuations pluviométriques sur la recharge des nappes qui conditionne la disponibilité de l'eau. 


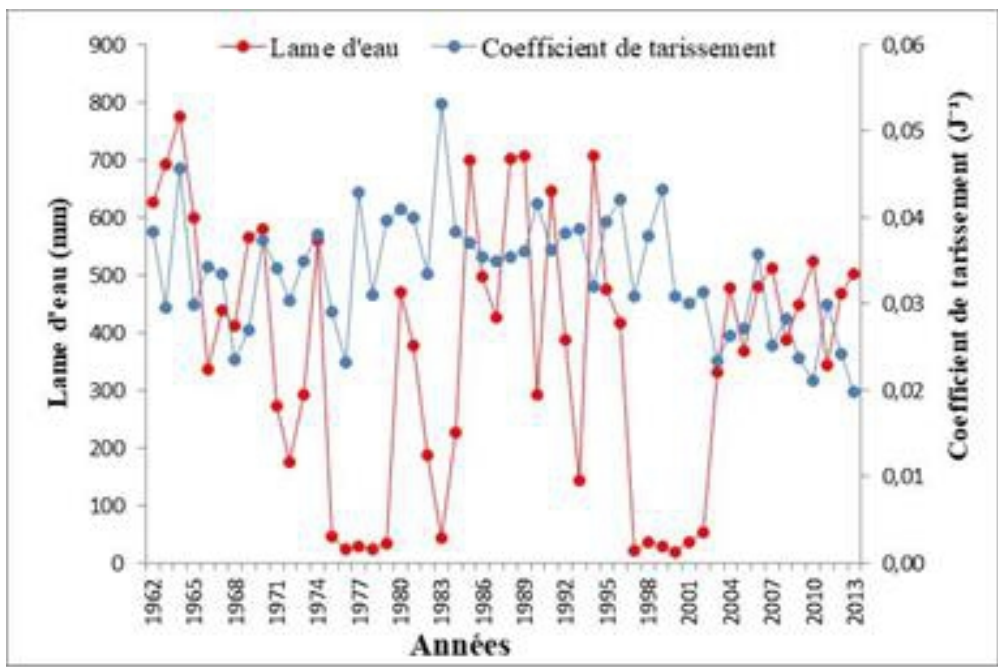

Figure 7: Évolution de la lame d'eau et du coefficient de tarissement du bassin versant du Bou à Tortiya au cours de la période 1962-2013

\subsection{Impact du climat sur les eaux souterraines du bassin du Bandama à Tortiya}

Les paramètres du bilan hydrologique calculés à la station de Sirasso et de Dikodougou de la zone d'étude selon la méthode de Thornthwaite sont consignés dans les tableaux 7 et 8 .

Tableau 7 : Bilan hydrique à la station de Sirasso (1901-2016)

\begin{tabular}{lcccc}
\hline Période & $\mathrm{P}(\mathrm{mm})$ & ETR $(\mathrm{mm})$ & $\mathrm{R}(\mathrm{mm})$ & $\mathrm{I}(\mathrm{mm})$ \\
\hline Chronique entière (1901-2016) & 1351 & 1012 & 95 & 244 \\
Avant rupture (1901-1971) & 1406 & 1037 & 98 & 271 \\
Après rupture (1972-2016) & 1266 & 988 & 88 & 190 \\
\hline
\end{tabular}

Tableau 8 : Bilan hydrique à la station de Dikodougou (1901-2016)

\begin{tabular}{lllll}
\hline Période & $\mathrm{P}(\mathrm{mm})$ & ETR $(\mathrm{mm})$ & $\mathrm{R}(\mathrm{mm})$ & $\mathrm{I}(\mathrm{mm})$ \\
\hline Chronique entière (1901-2016) & 1311 & 990 & 170 & 151 \\
& & & & \\
Avant rupture (1901-1970) & 1367 & 1017 & 178 & 172 \\
Après rupture (1971-2016) & 1224 & 964 & 160 & 100 \\
\hline
\end{tabular}

L'analyse du tableau 7 montre qu'à la station de Sirasso, les précipitations (P) moyennes estimées à 1406 mm avant la rupture (1901-1971) contribue à l'alimentation moyenne de l'écoulement (R) à hauteur de $98 \mathrm{~mm}$ soit environ $6,97 \%$ de la lame d'eau précipitée sur le bassin. Elles assurent également la recharge moyenne des aquifères du bassin par une lame d'eau infiltrée (I) d'environ $271 \mathrm{~mm}$ soit $19,27 \%$ des précipitations. A la rupture pluviométrique (1972-2016), les précipitions moyennes sont estimées à 1266 $\mathrm{mm}$. La lame d'eau ruisselée est de $88 \mathrm{~mm}$ soit $6,95 \%$ des précipitations. 
Quant à la lame d'eau infiltrée, elle est de $190 \mathrm{~mm}$ soit $15 \%$ des précipitations. La perte d'eau par évapotranspiration réelle (ETR) est estimée à 73,75\% avant la rupture et à 70,27\% après la rupture.

A la station de Dikodougou, avant la rupture pluviométrique (19011970) les précipitations moyennes estimées à 1367 mm contribuent à 178 mm de la lame d'eau ruisselée soit 13,02\% des précipitations. La lame d'eau infiltrée au cours de cette période est de $172 \mathrm{~mm}$ soit $12,58 \%$ des précipitations. Après la rupture pluviométrique (1971-2016), la lame d'eau ruisselée et infiltrée est passée respectivement à $160 \mathrm{~mm}$ soit 13,07\% des précipitations et $100 \mathrm{~mm}$ soit 8,17\% des précipitations.

\section{Discussion}

\subsection{Baisse de la pluviométrie et déficit pluviométrique}

Les méthodes statistiques (tests de rupture, indices hydroclimatiques, etc.) s'accordent sur la réalité d'une importante baisse de la pluviométrie dans le bassin du Bandama à Tortiya. Cette baisse s'est manifestée par des ruptures au sein des séries chronologiques; identifiées en 1971 pour la station de Sirasso et en 1970 pour Dikodougou. Ces résultats obtenus sont en accord avec les travaux antérieurs réalisés en Afrique de l'Ouest et Centrale en général (Paturel et al., 1998; Servat et al., 1999 ; Liénou, 2007; Amoussou, 2010) et en Côte d'Ivoire en particulier ( Soro et al., 2011 ; Noufé, 2011 ; Soro, 2014 ; Traoré, 2016 ; Amani et al., 2017 ) qui soulignent une baisse des précipitations à partir des années 1970. Les dates de ruptures déterminées au cours de cette étude s'intègrent dans l'intervalle (1966 - 1971) définies par les études antérieures citées ci-dessus. Les déficits pluviométriques calculés par rapport aux années de rupture identifiées sont inférieurs à 20\%. Selon Servat et al. (1998), les déficits pluviométriques sont parfois inférieurs à $20 \%$ dans certaines localités de l'Afrique de l'Ouest. C'est le cas de la station pluviométrique de Sirasso et Dikodougou dans le bassin du Bandama à Tortiya avec $9 \%$ et $11 \%$. Les résultats obtenus dans le bassin sont en conformité avec les conclusions des travaux antérieurs (Brou, 1997 et 2005 ; Brou et al., 1998 ; Koita, 2010). Selon ces auteurs, l'amplitude du phénomène n'est pas uniforme. De plus, les déficits pluviométriques sont perceptibles pendant les saisons des pluies durant lesquelles on enregistre une diminution du nombre de jours de pluies.

\subsection{Impacts hydrologiques}

La station hydrométrique de Tortiya a été choisie pour suivre la variabilité hydrologique du bassin pour la longueur de sa série. Ainsi, la diminution des précipitations dans le bassin du Bandama à Tortiya a une forte incidence sur le régime du Bou, l'un des principaux affluents du Bandama dans la région du Poro. En effet, les séries hydrométriques recueillies à cette 
station, montrent que les débits des cours d'eau chutent également à partir de 1971. Le déficit hydrologique après la date de rupture est estimé à 66,5\%. Les années 1977 et 1993 sont les plus déficitaires avec des modules respectifs de $16,1 \mathrm{~m}^{3} / \mathrm{s}$ et $6,1 \mathrm{~m}^{3} / \mathrm{s}$. Ces résultats obtenus sont en accord avec les travaux de Servat et al. (1998) qui montre que la majorité des ruptures identifiées au sein des séries hydrométriques en Afrique de l'Ouest est localisée entre 1968 et 1972. De façon générale, les déficits d'écoulements sont rarement inférieurs à $30 \%$ et parfois supérieurs à 55 voire 60\% (Servat et $a l ., 1998$ ). Par contre nos travaux diffèrent de ceux de Soro (2014) à cette même station qui situe la date de rupture à 1974. Cette différence pourrait s'expliquer par la provenance des données (Direction de l'Hydrologie) et par la longueur des chroniques (19621994).

\subsection{Impacts sur les ressources en eau de surface et souterraine}

L'impact de la variabilité pluviométrique sur les ressources en eau de surface a été analysé par l'étude des relations pluie-débit et pluie-volume d'eau mobilisés par les aquifères. Sur la base de ces relations, il apparaît clairement que la variabilité hydrologique est calquée sur la variabilité pluviométrique (Valimba et $a l ., 2005$; Kouakou et al., 2011). En effet, l'évolution comparée des indices de pluies et de débits a montré une incidence directe des déficits pluviométriques sur l'évolution hydrologique du cours d'eau. La similarité des indices de pluies et de débits identifiés souligne la dépendance des écoulements à la variation des précipitations.

Le coefficient de tarissement moyen annuel (1962-2013) à la station de Tortiya est évalué à $3,333 \cdot 10^{-2} / \mathrm{j}$ avant la rupture en 1971 et $3,361 \cdot 10^{-2} / \mathrm{j}$ après la rupture. Ces valeurs moyennes sont légèrement inférieures à celle déterminée par Soro (2014) à cette même station qui est de 3,84.10 $/ \mathrm{j}$ de 1962-1994 avec année de rupture 1974. Cette différence pourrait être due à la longueur des chroniques et aux différentes méthodes de comblement des données. Ces résultats montrent que les coefficients de tarissement connaissent une augmentation dans le bassin du Bandama à Tortiya après 1970 ce qui correspond à une vidange précoce et plus rapide des nappes souterraines (Mahé et $a l$., 1998). Ce phénomène a été décrit par certains auteurs ivoiriens dont Savané et al. (2001) à la station de Flampleu sur le bassin du Cavally, Saley (2003) dans la région de l'Ouest montagneux et Kouassi et al. (2013) dans le bassin du N'zi. Dans le même ordre d'idée, Aké (2010) note une augmentation du coefficient de tarissement dans le bassin versant de la Mé à Alépé. Les coefficients de tarissement dans ce bassin varient entre 1,0655.10$2 / \mathrm{j}$ et $9,679810^{-2} / \mathrm{j}$ avant 1970 , identifiée comme l'année de rupture. Après 1970 , les coefficients de tarissement fluctuent entre $2,2234.10^{-2} / \mathrm{j}$ et $5,388.10^{-}$ $2 \mathrm{j}^{-1}$ avec une moyenne de 3,5179. 10 $/ 2 / \mathrm{j}$. Traoré (2016) a mis également en évidence une forte variation du coefficient de tarissement à la station de Mé à 
Lobo Akoudzin dans le bassin versant d'aghien d'une année à l'autre sur la période $1983-2012$. Il oscille entre $2,410^{-2} / \mathrm{j}$ et $12,810^{-2} / \mathrm{j}$, avec une moyenne de $510^{-2} / \mathrm{j}$.

Les réserves en eaux souterraines sont sensibles aux variations pluviométriques. En effet, les volumes d'eau mobilisés moyens évoluent de $0,840 \mathrm{~km}^{3}$ avant 1971 à $0,508 \mathrm{~km}^{3}$ après 1971 , soit une baisse de $24,6 \%$. Ces résultats indiquent une diminution des volumes mobilisés par les aquifères après 1971, du fait d'une baisse sensible des réserves souterraines. Les variations des différents volumes mobilisés sont liées aux fluctuations pluviométriques dans le bassin. En effet, les travaux de Le Loeuff et Marchal (1993) ont montré que le régime des cours d'eau ivoiriens dépend des variations pluviométriques et provient d'une alimentation à peu près exclusivement par les eaux de ruissellement et secondairement par l'élimination de l'eau des sols. Les volumes d'eau mobilisés par les aquifères du bassin connaissent donc une baisse liée en partie à la baisse pluviométrique mise en évidence par les indices de Nicholson. Il s'ensuit un déficit hydrologique prononcé de l'ordre de $66,5 \%$.

La comparaison des moyennes annuelles de la lame d'eau infiltrée avant et après la rupture pluviométrique par la méthode de Thornthwaite donne une idée de l'impact des modifications pluviométriques sur la recharge des aquifères.

À la station de Sirasso, une baisse de la recharge des nappes de 42,63\% a été enregistrée depuis la rupture pluviométrique de 1971. Un déficit de $72 \%$ a également été enregistré à la station de Dikodougou après la rupture pluviométrique de 1970.

\section{Conclusion}

L'analyse de la variabilité hydroclimatique sur les stations pluviométriques du bassin du Bandama à Tortiya (1901-2016) a montré une alternance de période humide, normale et sèche. Elle est marquée par une baisse pluviométrique qui a été mise en évidence par les indices de Nicholson à partir de 1971 (période sèche 1971-2016) à la station de Sirasso, et à partir de 1970 (période sèche 1970-2016) à la station de Dikodougou. Le déficit pluviométrique est de $9 \%$ à Sirasso et de $10 \%$ à Dikodougou. La baisse de la pluviométrie a eu pour effet une diminution des apports d'eau qui transitent dans les cours d'eau dans le bassin ainsi que les réserves en eau souterraines avec un déficit hydrologique de 66,5\% des débits du Bandama à Tortiya. Les déficits d'écoulement sont bien supérieurs à ceux des précipitations. Le coefficient de tarissement moyen annuel (1962-2013) à la station de Tortiya est évalué à $3,333 \cdot 10^{-2} / \mathrm{j}$ avant la rupture en 1971 et $3,361 \cdot 10^{-2} / \mathrm{j}$ après la rupture soit une augmentation de $0,84 \%$. Ces résultats mettent en évidence une augmentation du coefficient de tarissement après 1971, autrement dit une 
faible vidange des aquifères alimentant l'écoulement de base. Les volumes d'eau mobilisés par les aquifères varient entre $0,4222 \mathrm{~km}^{3}$ et $1,5985 \mathrm{~km}^{3}$ avant 1971. Après 1971 les volumes mobilisés par les aquifères fluctuent entre $0,02697 \mathrm{~km}^{3}$ et $1,3136 \mathrm{~km}^{3}$. Les volumes d'eau mobilisés moyen évoluent de $0,8404 \mathrm{~km}^{3}$ avant 1971 à $0,5082 \mathrm{~km}^{3}$ après 1971 soit une baisse de $65,4 \%$. Ces résultats mettent en évidence une diminution des volumes mobilisés par les aquifères après 1971. Ces variations du volume d'eau mobilisé par les aquifères suggèrent une régression considérable des réserves souterraines.

L'évaluation de la recharge calculée par la méthode de Thornthwaite sur la chronique (1901-2016) est de 244 mm à la station de Sirasso et de 151 $\mathrm{mm}$ à la station de Dikodougou soit un volume d'eau infiltré respectivement égal à $854000000 \mathrm{~m}^{3}$ et $528500000 \mathrm{~m}^{3}$. Le volume d'eau moyen infiltré est alors estimé à $691250000 \mathrm{~m}^{3}$.

Cette étude montre que l'alimentation en eau des aquifères du bassin versant du Bandama à Tortiya n'est pas très bien assurée à cause de la faiblesse des écoulements due à une baisse de la pluviométrie depuis les années 70.

\section{Remerciements}

Les auteurs de ce présent article remercient le PASRES (Programme d'Appui Stratégique à la Recherche Scientifique) et le CSRS (Centre Suisse de Recherches Scientifiques en Côte d'Ivoire) pour avoir accepté de financer ce projet de recherche. Ils remercient également la Direction Générale des Infrastructures de l'Hydraulique Humaine (DGIHH) pour avoir fourni les données hydrométriques et le Laboratoire des Sciences et Techniques de l'Eau et de l'Environnement (LSTEE) de l'Université Felix Houphouët Boigny pour l'accueil.

\section{References:}

1. Aké G. E. (2010). Impacts de la variabilité climatique et des pressions anthropiques sur les ressources hydriques de la région de Bonoua (SudEst de la Côte d'Ivoire). Thèse de l'Université Félix Houphouët Boigny de COCODY, $234 \mathrm{p}$.

2. Amani M. K. Adjoa V. S. A., Koffi E.K., Kouakou.B D., Koffi F.K., et Biemi. J. (2017 a). Analyse de la persistance de la sécheresse en Afrique de l'Ouest: caractérisation de la variabilité climatique récente en Côte d'Ivoire. Environmental and Water Sciences, Public Heath \& Territorial Intelligence, Vol. 1, ํ2, 47-59.

3. Amani M. K., Adjoa V. S. A., Koffi E.K., Kouakou.B D., Koffi F.K., et Biemi. J. (2017 b). Analyse des impacts hydrologiques de la variabilité climatique en Afrique de l'Ouest : cas du bassin versant du Bandama en Côte d'Ivoire. Larhyss Journal, N³1, 19-40. 
4. Amani M.K., N'guessan Bi M.T., Koffi F.K., Kassi A.K., Okaingni.J.C., et Biemi.J., (2012). Application de la méthode des simulations croisées à l'analyse de tendances dans la relation pluiedébit à partir du modèle GR2M: Cas du bassin versant du N'ziBandama (Côte d'Ivoire). Comptes Rendus de l'Académie des Sciences de Paris, Géoscience, Vol. 344, º5,288-296.

5. Amoussou E. (2010). Variabilité pluviométrique et dynamique hydrosédimentaire du bassin-versant du complexe fluvio-lagunaire Mono-Ahémé-Couffo (Afrique de l'Ouest). Thèse de Doctorat, Université de Bourgogne, 310 p.

6. Assoma. T.V. (2013). Variabilité hydroclimatique et évaluation des ressources en eau du bassin côtier de l'Agneby Sud-est de la Côte d'Ivoire : apport des outils statistiques, de la modélisation et de la télédétection optique et radar. Thèse de doctorat l'Université Félix Houphouët Boigny de COCODY, 197 p.

7. Brou Y.T. (2005). Climat, mutations socio-économiques et paysages en Côte d'Ivoire. Mémoire de l'Habilitation à Diriger des Recherches, Univ. Sci. Techn. Lille, France, 212 p.

8. Brou Y.T, Servat E. et Paturel J-E. (1998). Activités humaines et variabilité climatique: cas du Sud forestier ivoirien. Water Resources Variability in Africa during the XXth Century (Proceedings of the Abidjan'98 Conference held at Abidjan, Côte d'Ivoire. IAHS, N²52, pp. 365-373.

9. Brou Y.T. (1997). Analyse et dynamique de la pluviométrie dans le Sud forestier ivoirien : recherche de corrélations entre les variables climatiques et les variables liées aux activités anthropiques. Thèse 3ème cycle, Univ. Cocody, Côte d'Ivoire, 211 p.

10. Chuzeville B. (1990) : Hydrologie tropicale et appliquée en Afrique subsaharienne. Collection Maîtrise de l'eau, 269 p.

11. Djro S. C. (1998) : Évolution tectono-métamorphique des gneiss granulitiques archéens du secteur de Biankouma. Thèse de Doctorat d'Etat, Université d'Abidjan 171p.

12. FAO (2005). Global forest resources assessment 2005. Progress towards sustainable forest management. FAO Forestry Paper, (2006) 147.

13. GIEC. (2007). Contribution du groupe de travail 1 au 4ème rapport d'évaluation du GIEC. Bilan 2007 des changements climatiques : les bases scientifiques physiques, $25 \mathrm{p}$.

14. Jourda P. (2005). Méthodologie d'application des techniques de télédétection et des systèmes d'information géographiques à l'étude des aquifères fissurés d'Afrique de l'Ouest. Concept de hydrotechnique spatiale : cas des zones tests de la Côte d'ivoire. Thèse 
de Doctorat d'État ès Sciences Naturelles, Université de CocodyAbidjan, Côte d'ivoire, 398 p.

15. Kanohin F., Saley M.B. et Savané I. (2009). Impacts de la variabilité climatique sur les ressources en eau et les activités humaines en zone tropicale humide: cas de la région de Daoukro en Côte d'Ivoire. Revue European Journal of Scientific Research, vol. 26, n 2, pp. 209-222.

16. Koita M. (2010). Caractérisation et modélisation du fonctionnement hydrodynamique d'un aquifère fracturé en zone de socle. Région de Dimbokro-Bongouanou (Centre Est de la Côte d'Ivoire). . Thèse de Doctorat de l'Université Montpellier II, 236 p.

17. Kouakou K. E. (2011). Impacts de la variabilité climatique et du changement climatique sur les ressources en eau en Afrique de l'Ouest : Cas du bassin versant de la Comoé. Thèse de Doctorat de l'Université de Cocody (Abidjan-Côte d'Ivoire), 204 p.

18. Kouassi A.M., Kouame K.F., Saley M. B., et Biemi J. (2013). Impacts des changements climatiques sur les eaux souterraines des aquifères de socle cristallin et cristallophyllien en Afrique de l'Ouest : cas du bassin versant du N'zi-Bandama (Côte d'Ivoire). Larhyss Journal, N¹6, 121-138.

19. Kouassi A.M., Kouame K.F., Goula B.T.A., Lasm T., Paturel J.E. et Biemi J. (2008). Influence de la variabilité climatique et de la modification de l'occupation du sol sur la relation pluie-débit à partir d'une modélisation globale du bassin versant du N'zi (Bandama) en Côte d'Ivoire. Revue Ivoirienne des Sciences et Technologie, Vol.11, pp. 207-229.

20. Le Loeuff P. et Marchal E. (1993) : Géographie littorale, Environnement et ressources aquatiques de Côte d'Ivoire. Le milieu marin. 8p.

21. Liénou G. (2007). Impacts de la variabilité climatique sur les ressources en eau et les transports de matières en suspension de quelques bassins versants représentatifs au Cameroun. Thèse de Doctorat, Université de Yaoundé I, Cameroun, 405 p.

22. Mahé G, Dessouassi R., Bandia C. et Olivry J. C. (1998). Comparaison des fluctuations interannuelles de piézométrie, précipitation et débit sur le bassin versant du Bani à Douna au Mali. International Association of Hydrological Sciences, 252, pp.289-295.

23. Nicholson S. E. (1993). An overview of African's rainfall fluctuation of the last decade. International Journal of Climatology, vol.6, pp.1463-1466.

24. Nka.B.N. (2016). Contribution à l'actualisation des normes hydrologiques en relation avec les changements climatiques et Environnementaux en Afrique de l'Ouest. Thèse de doctorat en 
sciences et technologies de l'eau, de l'énergie et de l'environnement de l'Institut International d'Ingénierie de l'eau et de l'environnement (2iE) et de l'Université PIERRE et MARIE CURIE (UPMC), $241 \mathrm{p}$.

25. Noufé D. (2011). Changements hydroclimatiques et transformations de l'agriculture: l'exemple des paysanneries de l'Est de la Côte d'Ivoire. Thèse de Doctorat de l'Université de Paris 1, $356 \mathrm{p}$.

26. Paturel J. E, Servat E. et Delattre M. O. (1998). Analyse de séries pluviométriques de longue durée en Afrique de l'Ouest et Centrale non sahélienne dans un contexte de variabilité climatique. Journal des sciences hydrologiques, Vol.43, $\mathrm{N}^{\circ} 3$, pp. 937-945.

27. Paturel J. E. et Servat E. (1996). Procédure d'identification de «rupture» dans les séries hydrologiques; modification du régime pluviométrique en Afrique de l'ouest non sahélienne. In «L'hydrologie tropicale : géoscience et outil pour le développement ». IAHS, (238) $99-110$.

28. Pettitt A. N. (1979). A non-parametric approach to the change-point problem. Applied Statistics, 28 (2), pp.126-135.

29. Poilecot P., Bonfou K., Dosso H., Lauginie F., N'dri K., Nicole M., et Sangaré Y. (1991). Un écosystème de savane soudanienne : le Parc National de la Comoé (Côte d'Ivoire). Note technique 2 n'IVC/87/007, $346 \mathrm{p}$.

30. Saley M. B. (2003). Cartographie thématique des aquifères de fissures pour l'évaluation des ressources en eau. Mise en place d'une nouvelle méthode d'extraction des discontinuités images et d'un SIHRS pour la région semi-montagneuse de Man (Nord-Ouest de la Côte d'Ivoire). Thèse de Doctorat d'Université de Cocody-Abidjan, 209 p.

31. Savané I., Coulibaly K. M. et Gioan P. (2003). Étude comparative de trois méthodes de calcul du coefficient de tarissement des cours d'eau. Sécheresse, Vol.14, $\mathrm{N}^{\circ} 1$, pp.37-42.

32. Savané I., Coulibaly K.M. et Gioan P. (2001). Variabilité climatique et ressources en eaux souterraines dans la région semi-montagneuse de Man. Revue Sciences et changement planétaire-sécheresse, vol.12, $\mathrm{n}^{\circ} 4,237 \mathrm{p}$.

33. Savané I. (1997). Contribution à l'étude géologique et hydrogéologique des aquifères discontinus du socle cristallin d'Odienné (Nord-Ouest de la Côte d'Ivoire). Apport de la télédétection et des systèmes d'information hydrogéologique à référence spatiale (S.I.H.R.S.). Thèse de Doctorat d'Etat, es Sciences naturelles, Université d'Abidjan, $386 \mathrm{p}$.

34. Servat E, Paturel J.E. Lubes-Niel H. Kouamé B. Masson J.M. Travaglio M. et Marieu B. (1999). Différents aspects de la variabilité 
de la pluviométrie en Afrique de l'Ouest et centrale non sahélienne. Revue des sciences de l'eau, Vol.12, N², pp. 363-387.

35. Servat E., Paturel J. E., Kouame B., Travaglio M., Ouedraogo M., Boyer J. F., Lubes-Niel H., Fritsch J. M., Masson J. M. et Marieu B. (1998). Identification, caractérisation et conséquences d'une variabilité hydrologique en Afrique de l'Ouest et Centrale. International Association of Hydrological Sciences Publication, $\mathrm{n}^{\circ}$ 252, pp. 323-337.

36. Soro N. (2002) : Hydrochimie et géochimie isotopique des eaux souterraines du degré carré de Grand-Lahou et ses environs (sudouest de la Côte d'Ivoire). Implication hydrologique et hydrogéologique. Thèse de Doctorat d'Etat ès Sciences Naturelles, Université de Cocody ; 272p.

37. Soro T. D (2014). Évolution des ressources en eau du bassin versant du Haut Bandama à Tortiya (Nord de la Côte d'Ivoire) dans un contexte de variabilité et de changement climatiques : impacts hydrologiques, hydrogéologiques et hydrochimiques. Thèse de l'Université Félix Houphouët Boigny de COCODY, 276 p.

38. Soro T. D., Soro N., Oga Y. M. S., Lasm T. Soro G., Ahoussi K. E. et Biémi J. (2011). La variabilité climatique et son impact sur les ressources les ressources en eau dans le degré carré de Grand-Lahou (Sud-Ouest de la Côte d'Ivoire). Revue Géographie Physique et Environnement, vol. 5, pp. 55-73.

39. Touré S. (2007) : Pétrologie et géochronologie du massif granitoïde de Bondoukou Nord-Est de la Côte d'Ivoire. Évolution magmatique et contexte géodynamique au Protérozoïque inférieur. Relations avec le volcano-détritique du Zanzan, Koun, Tanda attribué au Tarkwaïen du Ghana. Implications paléogéographiques. Thèse de Doctorat d'Etat ès Sciences Naturelles, Université d'Abobo Adjamé, 434p.

40. Traoré A. (2016). Impacts des changements climatiques et du changement de l'occupation et de l'utilisation du sol sur les ressources en eau de l'environnement lagunaire d'aghien et de potou (sud-est de la Côte d'Ivoire). Thèse de l'Université Félix Houphouët Boigny de COCODY, 248 p.

41. Valimba P., Servat E., Mkhandi S.H. And Camberlin P. (2005) Spatiotemporal variations of seasonal and annual rainfall totals in Tanzania. Submit. to J. Hydrol. 12p. 